\title{
A late presentation of acute fatty liver of pregnancy resulting in massive obstetric haemorrhage during twin delivery managed with epidural analgesia.
}

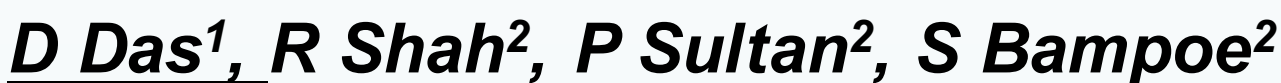 \\ 1. Princess Alexandra Hospital NHS Trust, Harlow, Essex, UK. \\ 2. Centre for Anaesthesia and Perioperative Medicine, University College London Hospitals NHS Foundation Trust, London, UK.
}

\section{Introduction}

Acute fatty liver of pregnancy (AFLP) is a life threatening condition with an incidence of 1:20,000. Aggressive, supportive management and early referral to intensive care (ICU) and a liver unit improves outcomes. We describe an unusual case of AFLP which presented without symptoms, diagnosed late in the second stage of labour. Immediate delivery with epidural analgesia was accompanied by massive obstetric haemorrhage $(\mathrm{MOH})$. Supportive management eventually led to full recovery.

\section{Background}

A 34-year-old primigravida presented to the obstetric unit for induction of labour (IOL) for DCDA twin pregnancy. 18 hours after IOL, an epidural was sited for analgesia. Initial blood results showed haemoglobin $(\mathrm{Hb}) 151 \mathrm{~g} / \mathrm{L}$ and platelets (PLT) of $89 \times 10^{9} / \mathrm{L}$. She was asymptomatic for a further 8 hours, but then became oliguric.

\section{Initial Investigations and Diagnosis}

Blood results showed PLT of $83 \times 10^{9} / \mathrm{L}$, urea $9.7 \mathrm{mmol} / \mathrm{L}$, creatinine $170 \mathrm{micromol} / \mathrm{L}$, INR 1.44 and elevated liver enzymes (ALT 370 IU/L, ALP 308 IU/L). An initial diagnosis of HELLP syndrome was made, $\mathrm{MgSO}_{4}$ infusion started and the patient was fluid restricted. Serum glucose was subsequently found to be low $(2.6 \mathrm{mmol} / \mathrm{L})$ and a revised diagnosis of AFLP was made.

\section{Obstetric and Anaesthetic Management}

As the patient's cervix was fully dilated, a multidisciplinary decision was made to perform a forceps delivery in theatre under epidural top-up. Coagulopathy was aggressively corrected with fibrinogen, fresh frozen plasma and cryoprecipitate. Following the establishment of invasive monitoring, forceps delivery of live twins was performed. Despite initial correction of clotting, blood tests in theatre showed worsening coagulopathy and acidosis. $\mathrm{MOH}$ with $\mathrm{3L}$ of blood loss followed requiring transfusion. Following transfer to ICU, blood results showed $\mathrm{Hb}$ of $93 \mathrm{~g} / \mathrm{L}$, PLT $57 \times 10^{9} / \mathrm{L}$, INR 1.22, urea $8.3 \mathrm{mmol} / \mathrm{L}$, creatinine $160 \mathrm{micromol} / \mathrm{L}$, bilirubin 63micromol/L and ALP 144IU/L. Fluid and blood product resuscitation continued. Liver ultrasound showed restricted blood flow in hepatic vein and inferior vena cava. Contrast CT was performed which showed distended hepatic veins, ascites and subcutaneous oedema consistent with liver failure.

\section{Recovery}

The patient improved steadily in ICU with conservative management under hepatology team. The epidural was removed under PLT cover and she was stepped down to ward after five days and discharged home on day seven. Liver and renal functions normalised after three weeks.

\section{Discussion}

AFLP is a rare disorder or pregnancy caused by impaired long chain fatty acid metabolism, which can progress rapidly to liver and renal failure. It is more common in in twin pregnancy and usually presents with nonspecific symptoms in third trimester or post-partum. This patient was asymptomatic with biochemical changes mimicking HELLP syndrome. Anaesthetic considerations include risk of regional block with coagulopathy balanced against complications of GA in the presence of encephalopathy and liver failure. Correction of coagulopathy and systemic stabilisation may be required to facilitate delivery and early, aggressive supportive treatment may reduce the risk of fulminant liver failure requiring transplant. 